\title{
ВЛИЯНИЕ ДЕФИЦИТА ВИТАМИНА D НА СЕРДЕЧНО-СОСУДИСТУЮ СИСТЕМУ
}

\section{INFLUENCE OF VITAMIN D DEFICIENCY ON THE CARDIOVASCULAR SYSTEM}

\section{O. Vengrzhinovskaya I. Bondarenko}

Summary. Vitamin D deficiency is diagnosed in about $30-50 \%$ of the world's population [1]. It should be noted that Vitamin D deficiency increases in proportion to the distance from the equator, which explains the increased filtration of UV rays. [2]. It causes not only chit, osteoporosis, osteomalacia, but also cardiovascular pathology. The Russian and foreign studies presented in this review prove that moderate and severe vitamin D deficiency is a risk factor for the development of cardiovascular diseases. [3].

A decrease in the level of vitamin $D$ in humans is a risk factor for cardiovascular pathology: arterial hypertension (AH), diabetes mellitus (DM), dyslipidemia, myocardial fibrosis and a predictor of adverse cardiovascular events — strokes and heart attacks.

It has been shown that vitamin $D$ has a protective effect on blood vessels, reducing endothelial dysfunction, has a positive effect on blood pressure, slows down vascular remodeling and myocardial fibrosis, reduces the risk of left ventricular hypertrophy and atherosclerosis, reduces insulin resistance, and affects the course of inflammatory processes [4].

Keywords: vitamin D deficiency, 25(OH)D, 1,25(OH)2D, cardiology, arterial hypertension, atherosclerosis, disturbance of heart rate, congestive heart failure.
Венгржиновская Оксана Игоревна

Врач-ординатор эндокринолог, ФГБУ

«Национальный медицинский исследовательский иентр эндокринологии» Министерства здравоохранения Российской Федерации, Москва vengrzhinovskay@gmail.com

Бондаренко Ирина Зиятовна

Врач-кардиолог, д.м.н., с.н.С., ФГБУ «Начиональный медицинский исследовательский чентр эндокринологии» Министерства здравоохранения Российской Федерачии, Москва

Аннотация. Дефицит витамина D встречается примерно у 30-50\% населения мира [1]. Необходимо отметить, что дефицит Витамина D, растет пропорционально расстоянию от экватору, что объясняет увеличение фильтрации УФ-лучей. [2]. Он вызывает не только -рахит, остеопороз, остеомаляцию, но и сердечно-сосудистую патологию. Проведенные российские и зарубежные исследования, представленные в данном обзоре, свидетельствуют о том, что умеренная и тяжелая недостаточность витамина D является фактором риска развития сердечно-сосудистых заболеваний. [3].

Снижение уровня витамина D у человека является фактором риска сердечно-сосудистой патологии: артериальной гипертензии (АГ), сахарного диабета (СД), дислипидемии, фиброза миокарда и предиктором неблагоприятных сердечно-сосудистых событий - инсультов и инфарктов.

Было показано, что витамин D оказывает вазопротекторный эффект снижая эндотелиальную дисфункцию, положительно влияет на артериальное давление, замедляет ремоделирование сосудов и фиброз миокарда, снижает риск развития гипертрофии левого желудочка и атеросклероза, уменьшает резистентность к инсулину, и влияет на течение воспалительных процессов [4].

Ключевые слова: дефицит витамина D, 25(OH)D, 1,25(ОН)2D, кардиология, атеросклероз, артериальная гипертензия, нарушение сердечного ритма, $\mathrm{XCH}$.

и витамин D превращается в 25(OH)D (25-гидроксивитамин D) (кальцидиол), далее второй этап гидроксилирования в почках - образуется активная форма витамина $\mathrm{D}-1,25(\mathrm{OH}) 2 \mathrm{D}$ (1,25-дигидроксивитамин D).

Спектр действия витамина D не ограничивается только контролем кальций-фосфорного обмена, он влияет и на развитие сердечно-сосудистых заболеваний. Что было подтверждено в масштабном исследовании Framingham Offspring Study, которое длилось более 
5 лет. Было показано, что частота сердечно-сосудистых событий (ишемии, инфарктов, инсультов) была выше на 33\% в группе пациентов с дефицитом витамина D, а также была показана прямая корреляция с уровнем витамина D и наличием артериальной гипертензии [5]

Витамин D влияет не только на течение и прогноз хронических заболеваний, но и на острые состояния. Среди пациентов, поступивших в отделения интенсивной терапии (ОИТ), у 40-70\% встречается дефицит витамина D. По данным систематического обзора 14 исследований (в которых участвовали 9715 пациентов ОИТ) выявлено, что дефицит витамина D был ассоциирован с более высокой смертностью. Также в обзоре сделан вывод, что дефицит витамина D может быть предиктором неблагоприятных последствий для здоровья среди пациентов ОИТ [6].

По результатам еще одного крупного исследования, установили, что у пациентов с низконормальным уровнем 25(OH)D (<37.5 нмоль/л), в сравнении с пациентами с достаточными уровнями ( $\geq 75$ нмоль/л) риск развития ИМ повышался более чем в 2 раза [7].

Исходя из вышеуказанных исследований, дефицит витамина D, является одним из факторов риска развития либо ухудшения течения сердечно-сосудистых заболеваний. Далее будет рассмотрено влияние дефицита витамина D на конкретные сердечно-сосудистые патологии.

\section{$\triangle$ ефицит витамина D и развитие атеросклероза}

Развитие атеросклероза является серьезным фактором риска неблагоприятных сердечно-сосудистых исходов, но несмотря на обширное количество клинических исследований его патогенез до сих пор до конца не изучен.

Основным регулятором сосудистого гомеостаза является эндотелий. В результате эндотелиальной дисфунции происходят воспалительные реакции, которые могут привести к пролиферации гладких мышц, тромбогенезу и способствуют развитию атеросклероза. Витамин D играет протективную роль и снижает риск развития атеросклероза посредством: уменьшении адгезиии и агрегации тромбоцитов, снижения окислительного стресса, увеличении продукции NO, подавления высвобождения провоспалительых цитокинов и ингибирования пролиферации гладкомышечных волокон [3].

Ригидность сосудистой стенки - важный фактор в развитии атеросклероза. По данным исследований, у пациентов с уровнем 25(OH)D менее 20 нг/мл повышена скорость пульсовой волны в аорте> 9 м/сек ( $\mathrm{N}=4-$ 6 м/сек), при этом увеличение скорости пульсовой волны имеет прямую корреляцию с увеличением риска развития атеросклероза. В этом исследовании был сделан вывод - чем ниже уровень витамина D, тем больше жесткость артерий, при этом поддержание уровня витамина D в референсном интервале способствует 2-х кратному снижению риска развития атеросклероза [8]. Один из вероятных механизмов патогенеза - положительное влияние активного метаболит витамина D - 1,25(OH)2D. Он снижает отложение минеральных депозитов на эндотелии, регулируя содержание в сыворотке крови кальция и фосфора.

Одну из основных ролей в развитии атеросклероза играют воспалительные реакции. Наиболее широко изученными маркерами сосудистого воспаления являются С-реактивный белок (СРБ) и фактора некроза опухоли-а (ФНО- а). Большое количество исследований подтверждают, что уровень витамина D способен подавлять высвобождения ФНО- a, также уровень витамина D обратно пропорционален концентрации в сыворотке СРБ. Помимо этого высокий уровень витамина D достоверно ассоциирован с высокой концентраций интерлейкина-10, который обладает кардиопротективным действием, подавляя продукцию провоспалительных цитокинов [9].

Статины - основной компонент терапии атеросклероза. Существуют публикации, рекомендующие совместный прием витамина D со статинами. Витамин D ингибирует ГМГ-КоА-редуктазу, фермент играющий ключевую роль в развитии атеросклероза, тем самым усиливая терапевтическое действие статинов.

\section{Аефицит витамина D и развитие АГ}

Взаимосвязь дефицита витамина D и развития артериальной гипертензии обсуждалась еще с конца прошлого века. Первое исследование, выявившее их взаимосвязь - Rostand в 1979г, было показано, что риск развития АГ выше у людей с дефицитом витамина $D$ (живущих дальше от экватора). В исследовании по изучению ультрафиолетового облучения (УФО) также было подтверждено влияние УФО на артериальное давление (АД). После воздействия УФО наблюдалось умеренное, статистически достоверное, снижение систолического АД. [10].

Существует несколько механизмов действия витамина D на уровень АД. Важным компонентом в контроле артериального давления, водно-солевого обмена и сосудистого тонуса является ренин-ангиотензин-альдостероновая система (РААС). По данным послед- 
них исследований активный метаболит витамина D $-1,25(\mathrm{OH}) \mathrm{D}$ участвует в регуляции РАAC, подавляя экспрессию гена ренина. Данное влияние было доказано в исследовании на грызунах: ингибирование синтеза 1,25(OH)D приводит к повышению экспрессии ренина и наоборот - введение грызунам 1,25(OH)D подавляет экспрессию ренина. Второй механизм - это снижение экспрессии эндотелиальной NO-синтетазы при дефиците витамина D, что ведет к повышению жесткости сосудистой жесткости и развитию эндотелиальной дисфункции. При этом введение 1,25(OH)2D оказывает протективное действие - увеличивает выработку NO в эндотелии [11].

Риск развития АГ у пациентов с дефицитом витамина D оценивалось во многих исследованиях. Два проспективных когортных исследования длительностью 16-18 лет, соответсвенно, с участием 38388 мужчин и 77531 женщины.Выявили, что риск развития АГ у мужчин с уровнем 25(OH) D менее 15 нг/мл значительно выше, чем при уровне 25(OH)D более 30 нг/мл, при анализе риска развития АГ у женщин также были получены достоверные различия [12]. Исследование NHANES III на 15000 пациентов также показало отрицательную корреляцию с уровнем витамина D и повышением цифр АД.

В качестве терапии АГ целесообразно добавление витамина D в комбинации с антигипертензивными препаратами, это приводи к снижению уровня систолического АД и и улучшению функции левого желудочка [13].

\section{Нарушение серАечного ритма и $А$ фицит витамина D}

Дефицит витамина D влияет также на сердечный ритм, однако патофизиологические механизмы этого влияния до конца еще не изучены.

В Шотландии проводилось 6-летнее исследование суточной вариабельности частоты госпитализации и смертности вследсвии фибрилляции предсердций (ФП). Было выявлено достоверное увеличение на $12 \%$ частоты госпитализаций в зимний период у женщин и достоверное увеличение смертности на $22 \%$ от ФП у мужчин [14]. В исследовании NHANES на 27153 пациентов в двух временных интервалах с 1988 по 1994 и с 2001 по 2006 год установили, что частота сердечных сокращений была достоверно выше на 2,1 уд/мин у у пациентов уровнем 25(OH)D (менее 10.0 нг/мл), чем у участников с более высоким уровнем 25(OH)D в референсном интервале. В отношении АД также отмечено повышение среднего АД в группе пациентов с уровнем 25(OH)D менее 10.0 нг/мл, в сравнении с пациентами с уровнем 25(OH)D15-35нг/мл.
Диаметр левого предсердия и давление в легочной артерии в значительной степени связаны с возникновением ФП. По данным проведенного исследования выявили, что уровень витамина D коррелирует с данными показателями у пациентов с неклапанной персистирующей ФП [15]. Мета-анализ 8 исследований с участием 27307 пациентов также выявил, что дефицит витамина $\mathrm{D}$ является одним из предиктором развития ФП [16].

Учитывая проведенные исследования дефицит витамина $D$ играет немаловажную роль в развитии нарушений сердечного ритма.

\section{Аефичит витамина D и хроническая серлечная неАостаточность}

Витамин D имеет многофакторное влияние на развитие хронической сердечной недостаточности (ХCH). Одним из факторов является развитие вторичного гиперпаратиреоза вследствие дефицита витамина D. Высокий уровень паратиреоидного гормона (ПТГ) провоцирует кальцификацию сердечных клапанов, тем самым увеличивая риск развития ХСН. Следующий фактор - это подавление при нормальных уровнях 1,25(ОН)2D синтеза провоспалительных цитокинов: ИЛ-1, ИЛ-6, ИЛ-8 и ФНО-а, что в свою очередь снижает частоту развития XCH [17]. Также немаловажный фактор развития $\mathrm{XCH}$ - регуляция витамином D активности теломеразы, именно укорочение теломер одна из причин старения, которое увеличивает риск развития $\mathrm{XCH}$ и других возраст-ассоциированных заболеваний[18].

B отношении дефицита витамина D и развития XCH было проведено несколько масштабных исследований. Одно из них длилось 2,5 года и закончилось в 2016 году, была выявлена отрицательная корреляционная связь уровня витамина D с концентрацией NTproBN и фракцией выброса левого желудочка, данная корреляция была также подтверждена и в другом исследовании но помимо этого, продемонстрировали, что связаны дисфункция миокарда, и смертностью от сердечной недостаточности были достоверно связаны с низкими уровенями 25(OH)D и 1,25(OH)2D [19]. Способность витамина подавлять выработку провоспалительных цитокинови увеличивать выработку противовоспалительных была также подтверждена в исследовании на 123 пациентах. В течении 9-ти месяцев они получали терапию витамином D в дозировке 2000МЕ. При котроле после терапии было выявлено снижение ФНО-а и увеличение уровня противовоспалительного ИЛ-10 [20].

Исходя из выше проведенных исследований можно составить данную схему действия дефицита витамина 


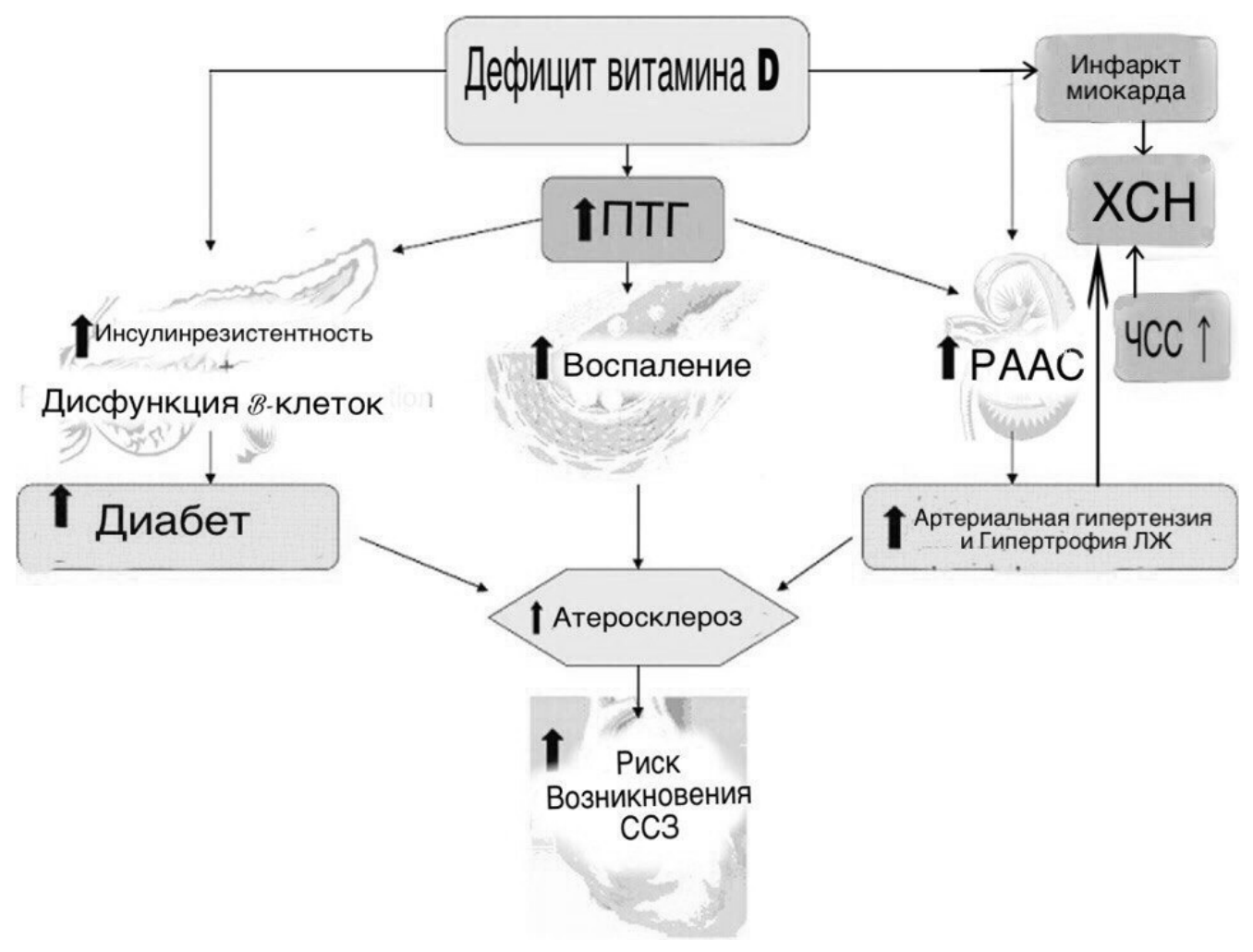

Рис. 1

D на сердечно-сосудистую систему. (Схема 1). ХCH -хроническая сердечная недостаточность; ПтГ - паратиреоидный гормон; ЧСС - частота сердечных сокращений; ЛЖ- левый желудочек; СС3 - сердечно-сосудистые заболевания (рис. 1).

Изучение эффективности применения витамина D установило его положительное влияние для профилактики и лечения сердечно-сосудистых заболеваний. Совокупность механизмов влияния витамина D на сердечно-сосудистую систему велика, но патогенез некоторых эффектов до сих пор остается неясен, что требует дальнейшего исследования.

\section{Профилактика и лечение Аефицита витамина D}

Широкий популяционный скрининг дефицита витамина $\mathrm{D}$ не рекомендуется российской ассоциации эндокринологов (РАЭ), но необходим в группах риска по его дефициту. К группам риска дефицита витамина D относятся: пациенты с недостаточной инсоляцией (маломобильные, с фотодерматитом, альбинизмом и по др. причинам), пациенты с хронической болезнью почек, с синдромом мальабсорбции, печеночной недостаточностью, с ожирением, пациенты длительно принимающие глюкокортикостероиды.
По данным из клинических рекомендации РАЭ: дефицитом витамина D обозначается концентрация в сыворотке 25(OH)D < 20 нг/мл, недостаточностью - концентрация 25(OH)D от 20 до 30 нг/мл, Рекомендуемыми целевыми значениями 30-60 нг/мл.

B целях профилактики дефицита витамина D лицам пациентам в возрасте 18-50 лет необходимо получать не менее 600-800 ME витамина D в сутки, пациентам старше 50 лет - не менее 800-1000 ME витамина D в сутки. При нарушении всасывания/метаболизма витамина D - необходимо увеличение суточной дозировки в 2-3 раза для данной возрастной группы. Принимая во внимание токсическое действие больших доз витамина D (более 10000 ME в сутки) без регулярного скрининга уровня витамина $D$, прием таких доз нецелесообразен.

Для лечения дефицита витамина D используют схемы насыщающей и поддерживающей дозы: при уровне $25(\mathrm{OH}) \mathrm{D}<20$ нг/мл в сыворотке, лечение начинают с суммарной насыщающей дозы колекальциферола 400 $000 \mathrm{ME}$, при уровне 25(ОН)D в сыворотке 20-29 нг/мл для лечения используют дозу колекальциферола - 200 000 ME. В зависимости от кратности приема насыщающая доза колекальциферола 400000 МЕ может быть достигнута приемом 200000 ME каждый месяц в течение 
2-х месяцев, либо по 50000 ME каждую неделю, либо по $7000 \mathrm{ME}$.

\section{Зак^ючение}

Дефицита витамина D широко распространен и влияет на течение и развитие множества сердечно-сосудистых заболеваний. Кардиологические пациенты с ХCH, ИБС, АГ как правило ведут малоподвижный образ жизни, реже бывают на солнце, из-за чего они входят в группу риска по дефициту витамина D. Экономически более выгодна профилактика витамина D, чем лечение последствий его дефицита. В качестве профилактики может быть использован не только прием профилактических дозировок и достаточная инсоляция, но и обогащение продуктов питания витамином D (в Финляндии с 2003 году используется обогащение пищевых продуктов витамина D, что фактически устранило его дефицит у населения). Для внедрения в клиническую практику назначения витамина D в комплексе с препаратами для терапии сердечно-сосудистых заболеваний, определения точной терапевтической дозировки для кардиологических пациентов необходимы дальнейшие рандомизированные контролируемые исследования.

\section{ЛИТЕРАТУРА}

1. Lips P. Worldwide status of vitamin D nutrition. J Steroid Biochem Mol Biol 2010; 121:297-300

2. Mithal A. Treatment of vitamin D deficiency. Endocrine case management ICE/END0 2014 Meet-th- professor, Endocrine society 2014 pp.37-39

3. Menezes A.R., Lamb M.C., Lavie C.J., DiNicolantonio J.J. Vitamin D and atherosclerosis. Curr Opin Cardiol 2014; 29(6):571-577

4. Tomson J., Emberson J., Hill M., et al. Vitamin D and risk of death from vascular and non-vascular causes in the Whitehall study and meta-analyses of 12000 deaths. Eur Heart J 2013; 34(18): 1365-74.

5. Wang T.J., Pencina M.J., Booth S.L., et al. (2008) Vitamin D deficiency and risk of cardiovascular disease. Circulation 117:503-51

6. de Haan K., Groeneveld A.B., de Geus H.R., et al. Vitamin D deficiency as a risk factor for infection, sepsis and mortality in the critically ill: systematic review and meta-analysis // Crit. Care. \2014. \18. \P. 660. https://doi.org/10.1186/s13054-014-0660-4.

7. Giovannucci E, Liu Y, Hollis BW, Rimm EB. 25-hydroxyvitamin D and risk of myocardial infarction in men: a prospective study. Arch Intern Med 2008; 168: $1174-1180$

8. Mayer 0 Jr, Filipovský J, Seidlerová J, Vaněk J, Dolejšová M, Vrzalová J, Cífková R. The association between low 25-hydroxyvitamin D and increased aortic stiffness. J Hum Hypertens. 2012 Nov; 26(11):650-5

9. Zittermann A, Dembinski J, Stehle P. Low vitamin D status is associated with low cord blood levels of the immunosuppressive cytokine interleukin-10. Pediatr Allergy Immunol 2004;15:242-6

10. Weber KT, Rosenberg EW, Sayre RM. Suberythemal ultraviolet exposure and reduction in blood pressure. The American Journal of Medicine 2004;117(4):281-2.

11. Norman P.E., Powell J.T. Vitamin D and Cardiovascular Disease. Circulation Research. 2014; 114:379-393. D0I: 10.1161/CIRCRESAHA.113.301241

12. Forman JP, Giovannucci E, Holmes MD, Bischoff-Ferrari HA, Tworoger HA, Willett WC, Curhan GC. Plasma 25-hydroxyvitamin D levels and risk of incident hypertension. Hypertension 2007 May;49(5):1063-9.

13. Nadir M.A., Szwejkowski B.R., Witham M.D. Vitamin D and cardiovascular prevention. Cardiovasc Ther 2010; 28(4): 5-12

14. Murphy NF, Stewart S, MacIntyre K, Capewell S, McMurray JJ. Seasonal variation in morbidity and mortality related to atrial fibrillation. International Journal of Cardiology 2004 Dec;97(2):283-8.

15. Cerit, Levent Bermuda triangle; heart failure, atrial fibrillation, and vitamin D deficiency. Journal of Cardiovascular Medicine. 2017 — Volume 18 — Issue $2-\mathrm{p} 121$

16. Zhiwei Zhang, BS; Yajuan Yang, BS; Chee Yuan Ng, MD; Dandan Wang, BS; JianlongWang, BS; Guangping Li, MD, PhD; Tong Liu, MD, Ph D. Meta-analysis of Vitamin D Deficiencyand Risk of Atrial Fibrillation. Clin. Cardiol. 2016. 39, 9, 537-543

17. Kamen D.L., Tangpricha V. Vitamin D and molecular actions on the immune system: modulation of innate and autoimmunity. J Mol Med 2010; $88: 441-50$

18. Драпкина 0.М., Шепель РН. Теломеры и хроническая сердечная недостаточность. Кардиология 2014; 54(4): 60-7

19. Pilz S., Marz W., Wellnitz B., Seelhorst U., Fahrleitner-Pammer A., Dimai H., Boehm B., Dobnig H. Association of vitamin D deficiency with heart failure and sudden cardiac death in a large cross-sectional study of patients referred for coronary angiography. J Clin Endocrinol Metab 2008; 93(10):3927-393

20. Schleithoff SS, Zittermann A, Tenderich G, Berthold H, Stehle P, Koerfer R. Vitamin D supplementation improves cytokine profiles in patients with congestive heart failure: a double-blind, randomized, placebo-controlled trial. The American Journal of Clinical Nutrition 2006 Apr;83(4):754-9. 